\title{
Multi-color carrier-envelope-phase stabilization for high-repetition-rate multi-pulse coherent synthesis
}

\author{
Richard A. McCracken, ${ }^{1, *}$ Ilaria Gianani, ${ }^{2}$ Adam S. Wyatt, ${ }^{2,3}$ and Derryck T. Reid ${ }^{1}$ \\ ${ }^{1}$ SUPA, Institute of Photonics and Quantum Sciences, Heriot-Watt University, Edinburgh EH14 4AS, UK \\ ${ }^{2}$ Clarendon Laboratory, University of Oxford, Parks Road, Oxford OX1 3PU, UK \\ ${ }^{3}$ Central Laser Facility, Science and Technology Research Council, Rutherford Appleton Laboratory, \\ Harwell, Oxford OX11 OQX, UK \\ *Corresponding author: r.a.mccracken@hw.ac.uk
}

Received August 24, 2014; revised February 17, 2015; accepted February 17, 2015; posted February 18, 2015 (Doc. ID 233030); published March 17, 2015

\begin{abstract}
Using a zero-offset carrier-envelope locking technique, we have synthesized an octave-spanning composite frequency comb exhibiting 132-attosecond timing jitter between the constituent pulses over a one-second observation window. In the frequency domain, this composite comb has a modal structure and coherence which are indistinguishable from those of a comb that might be produced by a hypothetical single mode locked oscillator of equivalent bandwidth. The associated phase stability enables the participating multi-color pulse sequences to be coherently combined, representing an example of multi-pulse synthesis using a femtosecond oscillator. (c) 2015 Optical Society of America

OCIS codes: (120.3940) Metrology; (190.4970) Parametric oscillators and amplifiers; (320.7090) Ultrafast lasers.

http://dx.doi.org/10.1364/OL.40.001208
\end{abstract}

Ultra-broadband optical frequency combs are important resources for emerging applications, including precision spectrograph calibration [1], quantum optics [2], and arbitrary pulse synthesis [3]. Optical waveforms coherently synthesized from carrier-envelope-phase (CEP) controlled parent pulses were recently reported using both Ti:sapphire [4] and optical parametric amplifiers [5] , as well as molecular modulation of a Raman resonance [ $[\underline{6}$, with the high peak powers and low repetition frequencies of the resulting pulses making them of principal interest in strong-field physics, while high repetition frequency synthesis has been reported using Erbium technology [7], albeit with a limited frequency bandwidth. Here, we demonstrate a new source of multi-color CEP stabilized pulses from a high repetition frequency femtosecond optical parametric oscillator (OPO) [8] which can be coherently combined to synthesize a composite frequency comb spanning the visible region and which exhibits only 132 attoseconds of mutual timing jitter over a one-second observation window, making the output essentially indistinguishable from that which would be generated by a single mode locked oscillator if a laser of such exceptional bandwidth existed.

The piecewise assembly of a composite frequency comb from a number of parent pulse sequences requires that their repetition frequencies and carrier-envelope offset (CEO) frequencies be locked to a common value $[9,10]$, and has been demonstrated between two mode locked lasers with the same [11] and different [12] gain media, circumventing the bandwidth limit imposed by their individual emission spectra, but requiring sub-cycle timing jitter to be achieved between them [13]. The parent oscillators must be synchronized in both repetition rate and CEO phase with extreme precision. Such stabilization requires complex locking electronics, and thus far demonstrations of pulse synthesis have been limited to combining parent pulses from only two individual oscillators.

In contrast to multiple-laser approaches, a synchronously pumped femtosecond OPO provides parent pulses of different wavelengths by means of simultaneous parametric, sum- and difference-frequency generation processes within the OPO cavity. These pulse sequences are intrinsically synchronized to one another and to their pump laser, itself a mode locked oscillator. Photonenergy conservation in $\chi(2)$ frequency conversion processes ensures that the CEO frequencies of the pump, signal, and idler combs generated by an OPO are related by $f_{\mathrm{CEO}}^{p}=f_{\mathrm{CEO}}^{s}+f_{\mathrm{CEO}}^{i}[\underline{14}]$, and CEO-frequency control in an OPO has been demonstrated in a number of embodiments [15-17]. Stabilization of the CEO and repetition frequencies is a necessary, but not sufficient, condition for achieving a multi-color phase-coherent composite comb because separate provisions are needed to ensure that the absolute phase fluctuations between separate comb components are kept well below $2 \pi$. The frequencies of the individual components can be selected via the phase matching conditions of the nonlinear crystal and extended through further intra-cavity doubling and sum-frequency generation, allowing for broadband tunability.

The parent pulse sequences for our experiment took the form of broadband pump ( $p, 800 \mathrm{~nm})$, secondharmonic signal $(2 s, 530 \mathrm{~nm})$, and pump-signal sumfrequency ( $p s, 456 \mathrm{~nm})$ pulses with tens-of-milliwatts level average power (0.5 nJ pulse energy) produced by a Ti:sapphire-pumped PPKTP femtosecond OPO operating at $100 \mathrm{MHz}$ (Fig. 1). The technique of zero-offset CEO locking [18] was used to set the CEO frequencies of the participating combs to zero, resulting in the creation of a composite comb in which all the comb lines lie on integer multiples of the repetition frequency.

Zero-offset locking achieving $f_{\mathrm{CEO}}^{p}=f_{\mathrm{CEO}}^{s}=f_{\mathrm{CEO}}^{i}=0$ was implemented by using two feedback loops to stabilize the heterodyne beats between a pump supercontinuum and the frequency-shifted pump + idler and second-harmonic signal pulses [19]. The coherence properties of this comb are critically determined by the mutual timing jitter of the pulses and the quality of the 


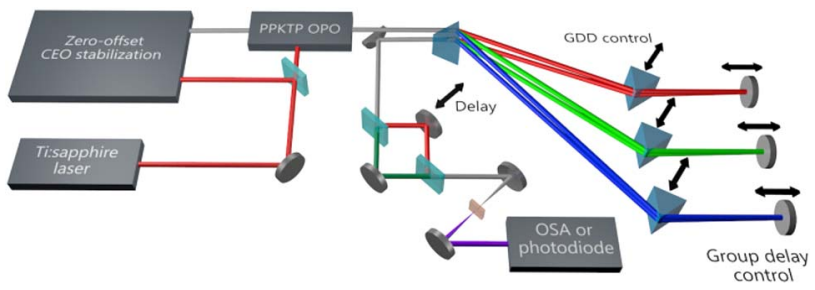

Fig. 1. Multi-pulse coherent synthesis and characterization scheme. Red ( $p, 800 \mathrm{~nm})$, green $(2 s, 530 \mathrm{~nm})$, and blue ( $p s$, $456 \mathrm{~nm}$ ) pulses from the OPO were separated in a prism-based compressor-delay line which allowed their phase, group-delay, and group-delay dispersion to be controlled. The pulses were adjusted to achieve temporal overlap and optimal compression in the downstream cross-correlator and XFROG apparatus used for characterization.

two CEO locks, which can introduce phase slips if they are insufficiently stable. Phase noise power spectral density (PSD) measurements for both locking loops (Fig. 2) show that over a one-second observation time (set by the lower frequency bound of the integrated cumulated phase noise), the accumulated phase-slips for both loops were less than $3 \%$ of an optical cycle, comparing well with previously published results [20] and indicating that residual CEO fluctuations are responsible for only a small amount of decoherence between the pulses.

The collinear pump, frequency-doubled-signal, and sum-frequency pump-signal beams from the OPO were steered into a quasi-common-path (QCP) prism delay line for both pulse compression and group-delay compensation (Fig. 1). The beams were dispersed by a fused silica prism then collected by three larger fused silica prisms, each positioned at a distance chosen to achieve optimal pulse compression at the subsequent measurement plane. Mirrors placed after each prism pair were used to form a folded four-prism compressor for each pulse; their positions were translated to compensate for the temporal walk-off between the pulses. A vertical tilt was added to pick off the returning beams, which were sent into a cross-correlation frequency-resolved optical gating (XFROG) apparatus [21].

The pump beam was separated from the visible pulses with a dichroic beam splitter and acted as the gate. The polarization of the visible pulses was rotated by

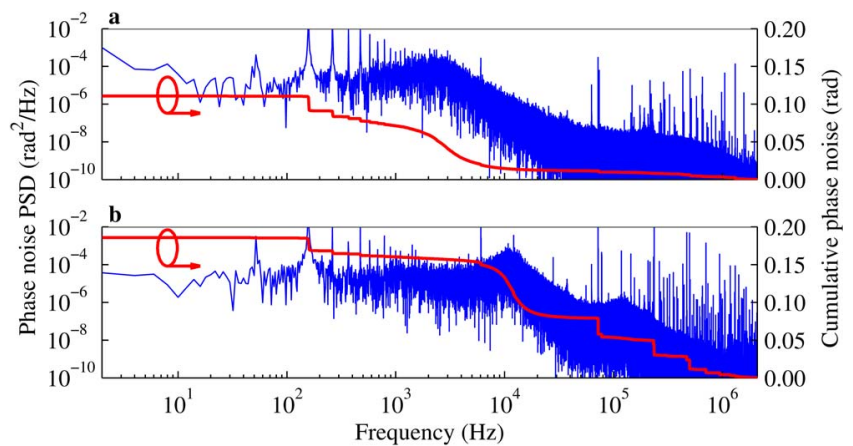

Fig. 2. In-loop phase noise power spectral density (PSD) with all CEO frequencies locked to zero. Phase noise PSD (blue) and cumulative phase noise (red) for (a) the idler and (b) the pumpsecond-harmonic signal CEO frequency. The cumulative phase noise integrated up to $1 \mathrm{MHz}$ is (a) $0.11 \mathrm{rad}$ and (b) $0.18 \mathrm{rad}$ over a one-second observation time. $90^{\circ}$ and recombined with the pump with a polarization beam splitting cube. An off-axis parabola focused the beams into a BBO crystal $[30 \mu \mathrm{m}$ BBO crystal cut at $45.6^{\circ}$ for Type II sum-frequency mixing (SFM)], and the SFM beams were reimaged using an aluminum spherical mirror into an ultraviolet optical spectrum analyzer (OSA, 227-450 nm). The $p, 2 s$, and $p s$ pulses were individually compressed in the prism delay line to approach their transform-limited durations (with higherorder dispersion terms resulting in a small residual chirp) and characterized in the XFROG; the pump was compressed first to ensure chirp-free gating pulses for the XFROG characterization of the visible pulses. The XFROG trace and corresponding retrieval data were used to obtain the relative delays between the pulses.

A balanced cross-correlator [22] was used to measure the mutual timing jitter between the pulses and was based on the XFROG apparatus described above, with the delay stage set at a fixed position and the ultraviolet OSA replaced with a silicon photodiode. Independent timing jitter measurements were performed between the $p$ and the $2 s$ pulses and the $p$ and $p s$ pulses. In each case, the delay was adjusted to a position where the SFM light generated in the BBO crystal varied linearly with small displacements of the delay stage. The photodiode signal was amplified and digitized using a 12-bit data acquisition card and a recorded calibration, which linearly mapped this signal to delay. The amplifier was used to fill the dynamic range of the acquisition card and to record and remove the average signal, ensuring that the recorded data represented the fluctuations about the mean value. Using the previous calibration, these intensity changes were mapped into delay fluctuations, providing a measure of the instantaneous timing jitter between the pump and the visible OPO pulses. The PSD of this timeseries provided the timing jitter frequency dependence, the integral over which gives the cumulative timing jitter over any chosen observation time.

Cross-correlation measurements between the $p, 2 s$, and ps pulses (Fig. 3) reveal how stabilizing the CEO of the resonant pulses reduces their jitter relative to the pump from 950 attoseconds - a substantial fraction of a carrier cycle - to 132 as over a one-second observation period. This reduction derives from the extreme

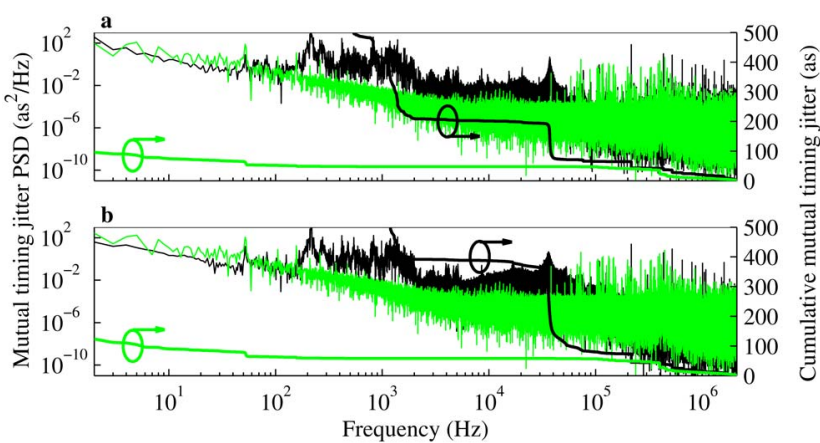

Fig. 3. Mutual timing jitter measurements. (a) Power spectral density and cumulative jitter between the pump and SHG signal pulses and (b) the pump and pump-signal SFM pulses when both CEO locking loops are unlocked (black) and locked (green). With both CEO loops locked, the cumulative mutual timing jitter between the pulses is 132 as. 
precision with which CEO locking also stabilizes the OPO center wavelength. Even in a common-path scheme, group-delay dispersion maps wavelength fluctuations into the time domain, with uncompensated GDD equivalent to $1 \mathrm{~mm}$ of silica causing a jitter of 250 as for a $1 \mathrm{~nm}$ variation of the shortest wavelength pulses. Consequently, active control of the OPO wavelength is critically important, and is achieved automatically in our scheme by the same feedback loops used to stabilize the CEO frequencies.

The cross-correlation is sensitive to contributions from both timing and intensity instabilities, so the recorded timing jitter $\tau_{\text {MEAS }}$ is composed of the true timing jitter $\tau_{\text {JTTER }}$ and the relative intensity noise (RIN) contribution to jitter $\tau_{\mathrm{RIN}}: \tau_{\mathrm{MEAS}}^{2}=\tau_{\text {ITTER }}^{2}+\tau_{\mathrm{RIN}}^{2}$. RIN measurements for the $p$ pulses were made by blocking the $2 s$ and $p s$ signals in combination with a band-pass filter. Similar measurements were made for the $2 s$ and $p s$ pulses. The voltage fluctuations measured in this way result from only RIN. We normalized these RIN fluctuations to quantify their contribution to the timing jitter measurement.

With the CEO locking off, the RIN contribution to the cumulative timing jitter was of the order of 50 as. When zero-offset CEO locking was established, the RIN timing jitter contribution for the pump and OPO pulses dropped to 8 as and 12 as respectively. The contribution of RIN fluctuations to the overall timing jitter was therefore small, the primary contribution arising from temporal drifts. Controlling the CEO frequencies of the pump and the OPO to establish both phase and wavelength stability remains the primary factor that determines the mutual timing jitter between the overlapping pulses.

An XFROG measurement of the composite frequency comb (Fig. 4) shows that the pulses are temporally overlapped and nearly transform limited after the QCP delay line. The retrieved temporal profile (Fig. 4(d)) is composed of a sequence of several high-contrast 2.2 fs pulses on a 39 fs duration pedestal. Such a pulse train could find applications in highly nonlinear phenomena, for example, high harmonic generation: the response due to the pulses other than at the peak are sufficiently suppressed, which, coupled with spectral filtering, can enable the generation of isolated attosecond pulses [23]. Alternatively, the pulse train can be utilized in stroboscopic style experiments [24] or coherent control of periodic dynamics [25], whereby the delay between adjacent pulses can be tuned to match the process via frequency selection of the individual pulses through the phase matching conditions of the OPO.

The pulses in the train are in phase, as demonstrated collectively by our jitter and CEO measurements. The relative phase of the parent pulses can be readily adjusted by sub-wavelength changes to their relative optical delays. However, XFROG is agnostic to this phase difference and cannot measure it; we have assumed zero relative phase for the composite pulse reconstruction. Potentially, spectral interferometry between the pulses and a pump supercontinuum could reveal this phase difference at a given target location.

The results presented here are not an intrinsic limitation of the setup described, but rather an embodiment of a specific permutation of the OPO cavity optics, nonlinear crystal, and characterization method. The CEP
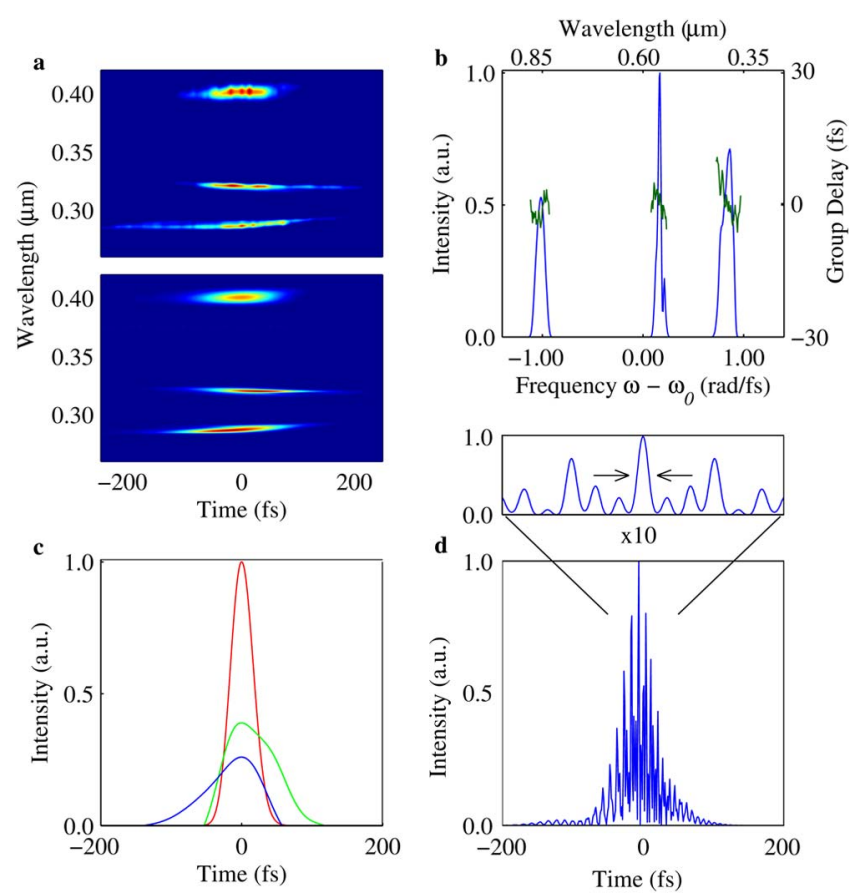

Fig. 4. XFROG retrieval after pulse compression and delay compensation. (a) Measured (top) and retrieved (bottom) XFROG traces. (b) Retrieved spectral intensity and phase profiles, showing pulse compression to near-transform-limited durations. (c) Individual temporal pulse intensities, obtained by spectrally filtering the XFROG retrieval data, with retrieved durations of $38 \mathrm{fs}(p$, red), $100 \mathrm{fs}$ ( $2 s$, green), and $115 \mathrm{fs}$ ( $p s$, blue); transform limited durations of $37 \mathrm{fs}(p), 80 \mathrm{fs}(2 s)$, and 86 fs $(p s)$. (d) Simulated temporal profile of the combined pulses assuming zero relative phase at the location of the measurement. Pulse train consists of several high-contrast pulses with durations of around 2.2 fs (arrows, inset panel).

stabilized second-harmonic pump $(2 p, 400 \mathrm{~nm})$ and pump-idler sum-frequency ( $p i, 642 \mathrm{~nm}$ ) pulses were also accessible from the exit mirror of the OPO, and could be compressed in a similar manner as detailed above. Sumfrequency phase matching conditions in the selected characterization crystal precluded characterization of the $2 p$ pulses, which could be addressed by using an alternative technique such as DFG-XFROG [26]. The choice of poling period in the PPKTP crystal for parametric gain did not provide efficient phase matching for $p i$ generation; however, using cascaded [17] or chirped [27] grating periods can improve this efficiency, as well as allow the direct generation of other sum-frequency or harmonic outputs from the OPO.

In conclusion, by exploiting the natural synchronism of pulses from a femtosecond optical parametric oscillator, we have shown for the first time to the best of our knowledge how parent-pulse sequences of three different colors can be used to assemble a composite frequency comb, whose mode structure and coherence are indistinguishable from those associated with a single mode locked oscillator. Just as low-phase-noise ultrafast lasers provided the basis for high resolution dual-wavelength pump-probe techniques, the mutual timing jitter of much less than one optical cycle exhibited by this unique source creates new opportunities for nondegenerate phase-coherent pump-probe measurements, for example 
in resolving the coherent redistribution of energy among electrons in a complex molecule [28].

The ability to carrier-envelope stabilize and shape several multi-color pulses while maintaining a low jitter phase lock opens up the possibility of generating subcycle pulses to study and control electronic processes on attosecond time-scales [29], particularly in nanoplasmonics [30], where the low field strengths and high repetition rates are ideally suited. For example, collective electron dynamics can be controlled in surface plasmons [31] and strong field phenomena can be achieved via field enhancement in metal tips leading to field ionization of electrons and extreme ultraviolet (XUV) attosecond pulses via high harmonic generation [32]. Such systems only require peak field strengths of $10^{10}-10^{11} \mathrm{~W} / \mathrm{cm}^{2}$, obtainable with a few tens-of-milliwatts average power at $100 \mathrm{MHz}$, leading to a time-averaged electron/XUV flux that can be much higher than that achieved with high energy kilohertz repetition rate lasers systems simply because of the factor of $10^{5}$ increase in repetition rate.

We gratefully acknowledge funding from the UK Engineering and Physical Sciences Research Council (EPSRC) under grant number EP/H000011/1.

\section{References}

1. G. G. Ycas, F. Quinlan, S. A. Diddams, S. Osterman, S. Mahadevan, S. Redman, R. Terrien, L. Ramsey, C. F. Bender, B. Botzer, and S. Sigurdsson, Opt. Express 20, 6631 (2012).

2. O. Pinel, P. Jian, R. M. de Araújo, J. Feng, B. Chalopin, C. Fabre, and N. Treps, Phys. Rev. Lett. 108, 083601 (2012).

3. Z. Jiang, C.-B. Huang, D. E. Leaird, and A. M. Weiner, Nat. Photonics 1, 463 (2007).

4. A. Wirth, M. T. Hassan, I. Grguras, J. Gagnon, A. Moulet, T. T. Luu, S. Pabst, R. Santra, Z. A. Alahmed, A. M. Azzeer, V. S. Yakovlev, V. Pervak, F. Krausz, and E. Goulielmakis, Science 334, 195 (2011).

5. S.-W. Huang, G. Cirmi, J. Moses, K.-H. Hong, S. Bhardwaj, J. R. Birge, L.-J. Chen, E. Li, B. J. Eggleton, G. Cerullo, and F. X. Kärtner, Nat. Photonics 5, 475 (2011).

6. H.-S. Chan, Z.-M. Hsieh, W.-H. Liang, A. H. Kung, C.-K. Lee, C.-J. Lai, R.-P. Pan, and L.-H. Peng, Science 331, 1165 (2011).

7. G. Krauss, S. Lohss, T. Hanke, A. Sell, S. Eggert, R. Huber, and A. Leitenstorfer, Nat. Photonics 4, 33 (2009).

8. D. C. Edelstein, E. S. Wachman, and C. L. Tang, Appl. Phys. Lett. 54, 1728 (1989).

9. L.-S. Ma, R. K. Shelton, H. C. Kapteyn, M. M. Murnane, and J. Ye, Phys. Rev. A 64, 021802 (2001).
10. R. K. Shelton, S. M. Foreman, L.-S. Ma, J. L. Hall, H. C. Kapteyn, M. M. Murnane, M. Notcutt, and J. Ye, Opt. Lett. 27, 312 (2002).

11. R. K. Shelton, L. S. Ma, H. C. Kapteyn, M. M. Murnane, J. L. Hall, and J. Ye, Science 293, 1286 (2001).

12. A. Bartels, N. R. Newbury, I. Thomann, L. Hollberg, and S. A. Diddams, Opt. Lett. 29, 403 (2004).

13. J. A. Cox, W. P. Putnam, A. Sell, A. Leitenstorfer, and F. X. Kärtner, Opt. Lett. 37, 3579 (2012).

14. J. Sun, B. J. S. Gale, and D. T. Reid, Opt. Express 15, 4378 (2007).

15. Y. Kobayashi, H. Takada, M. Kakehata, and K. Torizuka, Opt. Lett. 28, 1377 (2003).

16. J. Sun, B. J. S. Gale, and D. T. Reid, Opt. Lett. 32, 1396 (2007).

17. J. Sun and D. T. Reid, Opt. Lett. 34, 854 (2009).

18. R. A. McCracken, J. Sun, C. G. Leburn, and D. T. Reid, Opt. Express 20, 16269 (2012).

19. T. I. Ferreiro, J. Sun, and D. T. Reid, Opt. Lett. 35, 1668 (2010).

20. T. I. Ferreiro, J. Sun, and D. T. Reid, Opt. Express 19, 24159 (2011).

21. S. Linden, H. Giessen, and J. Kuhl, Phys. Status Solidi B 206, 119 (1998).

22. T. R. Schibli, J. Kim, O. Kuzucu, J. T. Gopinath, S. N. Tandon, G. S. Petrich, L. A. Kolodziejski, J. G. Fujimoto, E. P. Ippen, and F. X. Kärtner, Opt. Lett. 28, 947 (2003).

23. G. Sansone, E. Benedetti, F. Calegari, C. Vozzi, L. Avaldi, R. Flammini, L. Poletto, P. Villoresi, C. Altucci, R. Velotta, S. Stagira, S. De Silvestri, and M. Nisoli, Science 314, 443 (2006).

24. J. Mauritsson, P. Johnsson, E. Mansten, M. Swoboda, T. Ruchon, A. L'Huillier, and K. J. Schafer, Phys. Rev. Lett. 100, 073003 (2008).

25. M. P. A. Branderhorst, P. Londero, P. Wasylczyk, C. Brif, R. L. Kosut, H. Rabitz, and I. A. Walmsley, Science $\mathbf{3 2 0}$, 638 (2008).

26. S. Linden, J. Kuhl, and H. Giessen, Opt. Lett. 24, 569 (1999).

27. K. A. Tillman, D. T. Reid, D. Artigas, J. Hellström, V. Pasiskevicius, and F. Laurell, J. Opt. Soc. Am. B 20, 1309 (2003).

28. J. L. Herek, W. Wohlleben, R. J. Cogdell, D. Zeidler, and M. Motzkus, Nature 417, 533 (2002).

29. M. Schultze, E. M. Bothschafter, A. Sommer, S. Holzner, W. Schweinberger, M. Fiess, M. Hofstetter, R. Kienberger, V. Apalkov, V. S. Yakovlev, M. I. Stockman, and F. Krausz, Nature 493, 75 (2013).

30. S. Kim, J. Jin, Y.-J. Kim, I.-Y. Park, Y. Kim, and S.-W. Kim, Nature 453, 757 (2008).

31. M. I. Stockman, M. F. Kling, U. Kleineberg, and F. Krausz, Nat. Photonics 1, 539 (2007).

32. M. Krüger, M. Schenk, and P. Hommelhoff, Nature 475, 78 (2011). 